\title{
The Effect of Positive Airway Pressure Therapy on Sexual Life and Quality of Life in Women with Moderate or Severe Obstructive Sleep Apnea Syndrome
}

\author{
Dasdemir Ilkhan Gulay ${ }^{1, *}$, Celikhisar Hakan²
}

\author{
${ }^{1}$ Okmeydani Trainning and Research \\ Hospital, Chest Diseases Clinic Kaptan \\ Pasa Mahallesi, Istanbul, Turkey \\ ${ }^{2}$ Izmir Metropolitan Municipality \\ Hospital, Chest Diseases Clinic Gaziler \\ Caddesi, Izmir, Turkey \\ *Correspondence \\ gdasdemir1111@gmail.com \\ (Dasdemir Ilkhan Gulay)
}

\begin{abstract}
Background and Aim: Obstructive sleep apnea syndrome (OSAS) negatively affects quality of life and causes erectile dysfunction in men. However, there are few studies examining the effects of OSAS on women's sexual health. This study used validated measurement tools to evaluate whether the presence and severity of OSAS had an impact on female sexual function and if there was a change after 6 months of CPAP treatment. Scores were compared before and after CPAP in the same and between groups. Material and Method: The patients were divided into moderate and severe groups based on the Apnea-Hypopnea Index (AHI). The female patients included in the study were administered the Female Sexual Function Index (FSFI), and the Calgary sleep apnea quality of life index (SAQLI) questionnaires to evaluate their sexual function. After CPAP treatment for 6 months, FSFI, and SAQLI questionnaires were compared with the results before treatment. Results: Among study participants, 72 had severe and 76 had moderate OSAS. The mean age (standard deviation or SD) in the severe OSAS group was $40.97 \pm 5.65$ years and in the moderate OSAS group was $35.46 \pm 5.43$ years $(\mathrm{p}<$ 0.001 ). Body mass indexes of the severe and moderate OSAS groups were $33.10 \pm 3.33$ $\mathrm{kg} / \mathrm{m}^{2}$ and $31.04 \pm 3.42 \mathrm{~kg} / \mathrm{m}^{2}$, respectively $(\mathrm{p}<0.001)$. The mean Epworth scores $(\mathrm{SD})$ of the severe and moderate OSAS groups were $11.45 \pm 3.27$ and $9.52 \pm 3.31$, respectively $(\mathrm{p}=0.001)$. Based on FSFI scores, in the pre-treatment period, desire, lubrication, and satisfaction were significantly better in the moderate OSAS group. After treatment, only desire and satisfaction were significantly better in the moderate OSAS group. In both groups, after treatment, all parameters of the FSFI score were significantly better compared with the pretreatment values $(p=0.001)$. When SAQLI scores were compared, in the pre-treatment period, all parameters were significantly better in the moderate OSAS group. In both groups, after treatment, all parameters of the SAQLI score were significantly better compared with the pre-treatment values $(\mathrm{p}=$ 0.001). Conclusion: In women with severe and moderate OSAS, sexual functions and satisfaction levels were negatively associated with OSAS severity. Sexual functions and quality of life were significantly improved with effective treatment of OSAS.
\end{abstract}

\section{Keywords}

Obstructive sleep apnea syndrome, Polysomnography, Positive airway pressure therapy, Women, Sexual life

\section{Introduction}

Obstructive sleep apnea syndrome (OSAS) is characterized by obstruction of the upper airways with repetitive pauses in breathing during sleep causing excessive daytime sleepiness and impaired cognitive performance. Repeated episodes of airway occlusion during sleep results in hypoxemia and hypercapnia attacks increasing oxidative stress $[1,2]$. OSAS has many systemic effects that negatively affect quality of life. The data regarding sexual health in patients with OSAS is limited. Though there are some studies on male sexual health status, only a few studies are present about sexual functions in females with OSAS [3-6]. These studies reported that OSAS has negative impacts on sexual function in women independent of age and associated co-morbid diseases.

There are validated instruments to analyze sexual health status and quality of life in patients. In this study, we used validated instruments to evaluate whether the presence and severity of OSAS had an impact on female sexual function and whether there was a change after 6 months of CPAP treatment. 
TA B L E 1. Comparison of pretreatment and post-treatment FSFI scores between patients having moderate and severe OSAS.

\begin{tabular}{|c|c|c|c|}
\hline & Moderate OSAS (n:76) & Severe OSAS (n:72) & p1 \\
\hline \multicolumn{4}{|l|}{ Desire } \\
\hline Pre-treatment & $2.63 \pm 0.18$ & $2.45 \pm 0.52$ & 0.04 \\
\hline Post-treatment & $3.56 \pm 0.96$ & $3.82 \pm 0.85$ & 0.04 \\
\hline p2 & 0.001 & 0.001 & \\
\hline \multicolumn{4}{|l|}{ Arousal } \\
\hline Pre-treatment & $2.65 \pm 0.28$ & $2.58 \pm 0.51$ & 0.26 \\
\hline Post-treatment & $3.72 \pm 0.96$ & $4.11 \pm 0.74$ & 0.09 \\
\hline p2 & 0.001 & 0.001 & \\
\hline \multicolumn{4}{|l|}{ Lubrication } \\
\hline Pre-treatment & $3.63 \pm 0.31$ & $3.42 \pm 0.55$ & 0.08 \\
\hline Post-treatment & $4.82 \pm 0.74$ & $5.03 \pm 0.75$ & 0.08 \\
\hline p2 & 0.001 & 0.001 & \\
\hline \multicolumn{4}{|l|}{ Orgasm } \\
\hline Pre-treatment & $3.17 \pm 0.25$ & $2.99 \pm 0.69$ & 0.03 \\
\hline Post-treatment & $4.10 \pm 0.81$ & $4.08 \pm 1.00$ & 0.09 \\
\hline p2 & 0.001 & 0.001 & \\
\hline \multicolumn{4}{|l|}{ Satisfaction } \\
\hline Pre-treatment & $3.18 \pm 0.25$ & $3.01 \pm 0.46$ & 0.009 \\
\hline Post-treatment & $4.22 \pm 0.76$ & $3.58 \pm 0.99$ & $\mathbf{0 . 0 3}$ \\
\hline p2 & 0.001 & 0.001 & \\
\hline \multicolumn{4}{|l|}{ Pain } \\
\hline Pre-treatment & $3.74 \pm 0.41$ & $3.63 \pm 0.38$ & 0.09 \\
\hline Post-treatment & $4.28 \pm 0.75$ & $4.32 \pm 0.99$ & 0.9 \\
\hline p2 & 0.001 & 0.001 & \\
\hline \multicolumn{4}{|l|}{ Total score } \\
\hline Pre-treatment & $19.03 \pm 1.26$ & $18.10 \pm 2.33$ & 0.04 \\
\hline Post-treatment & $24.71 \pm 3.57$ & $25.67 \pm 7.09$ & 0.3 \\
\hline p2 & 0.001 & 0.001 & \\
\hline
\end{tabular}

p1: Comparison between moderate and severe OSAS patients; p2: Intra-group comparison between pre- and post-treatment values.

\section{Material and method}

This prospective study included premenopausal women with OSAS diagnosed with polysomnography. The local ethics committee approved the study and informed consent was obtained from the study participants. Patients with any neuropsychiatric disease that can alter the understanding and compilation of questionnaires were excluded from the study.

Demographic features including age, weight, and height were recorded. The patients were asked for their educational status. Smoking history and any chronic diseases was recorded. Body mass index (BMI) of the participants was calculated with the formula, BMI: weight/height ${ }^{2}$.

Patients were studied using identical, portable devices using a nasal pressure catheter, pulse oximetry, and thoracic and abdominal bands to record the nasal airflow, snoring, and respiratory movements (Alice 3; Healthdyne Technologies). Apneas were diagnosed as the cessation of breathing of $>$ 10 seconds. Hypopneas were diagnosed as the reduction in airflow $>30 \%$, associated with a desaturation of $\geq 4 \%$. The apnea and hypopnea index (AHI) was calculated as the total number of apneas and hypopneas, divided by the estimated sleep time, in hours. The patients were divided into two groups according to the Apnea-Hypopnea Index (AHI), as moderate (AHI between 15 and 30) and severe (AHI > 30) [7]. Epworth Sleepiness Scale scores were recorded in all patients before and after CPAP treatment [8].

Female Sexual Function Index (FSFI) is a scale consisting of six 6 different domains: desire, arousal, lubrication, orgasm, sexual satisfaction, and pain. A higher score means better function [9]. Calgary sleep apnea quality of life index (SAQLI) was developed as a sleep apnea specific quality of life tool. 
TA B L E 2. Comparison of pretreatment and post-treatment SAQLI scores between patients having moderate and severe OSAS.

\begin{tabular}{|lccc|}
\hline & Moderate OSAS (n:76) & Severe OSAS (n:72) & p1 \\
\hline Daily functioning & & & \\
\hline Pre-treatment & $3.48 \pm 0.58$ & $3.10 \pm 0.27$ & $\mathbf{0 . 0 0 1}$ \\
\hline Post-treatment & $5.27 \pm 1.15$ & $5.03 \pm 0.88$ & $\mathbf{0 . 1 7 6}$ \\
\hline p2 & 0.001 & 0.001 & \\
\hline Social interactions & & & $\mathbf{0 . 0 0 1}$ \\
\hline Pre-treatment & $3.43 \pm 0.74$ & $2.80 \pm 0.40$ & $\mathbf{0 . 0 0 1}$ \\
\hline Post-treatment & $5.10 \pm 1.20$ & $4.50 \pm 0.61$ & \\
\hline p2 & 0.001 & 0.001 & $\mathbf{0 . 0 0 1}$ \\
\hline Emotional functioning & & & $\mathbf{0 . 0 0 1}$ \\
\hline Pre-treatment & $3.25 \pm 0.64$ & $2.61 \pm 0.38$ & \\
\hline Post-treatment & $4.60 \pm 1.15$ & $4.03 \pm 0.85$ & \\
\hline p2 & 0.001 & 0.001 & $\mathbf{0 . 0 0 1}$ \\
\hline Symptoms & & & $\mathbf{0 . 0 0 1}$ \\
\hline Pre-treatment & $3.08 \pm 0.360$ & $1.88 \pm 0.50$ & $\mathbf{0 . 0 0 1}$ \\
\hline Post-treatment & $4.90 \pm 1.13$ & $3.33 \pm 0.46$ & $\mathbf{0 . 0 0 1}$ \\
\hline p2 & 0.001 & 0.001 & \\
\hline SAQLI score & $13.25 \pm 1.56$ & $10.42 \pm 0.91$ & \\
\hline Pre-treatment & $19.88 \pm 3.92$ & 0.001 & \\
\hline Post-treatment & 0.001 & & \\
\hline p2 & & & \\
\hline$p 1:$ int & & & \\
\hline
\end{tabular}

p1: Comparison between moderate and severe OSAS patients; p2: Intra-group comparison between pre- and post-treatment values.

It is a 35-item questionnaire that captures the negative effect of sleep apnea on 4 domains: daily functioning, social interactions, emotional state, and symptoms [10]. A higher score indicates a better quality of life. The SAQLI contains items that are important for patients with sleep apnea that have been designed as an outcome measures in clinical studies in sleep apnea after CPAP.

The patients included in the study were administered the FSFI, and SAQLI questionnaires to evaluate their sexual function and sleep. After CPAP treatment of 6 months, FSFI, and SAQLI questionnaires were repeated and compared with the results before treatment. Compliance of CPAP users was defined as patients with more than 4 hours of CPAP use per night for 4 weeks immediately before the 6th-month follow-up visit.

\subsection{Statistical analysis}

Statistical analyses were performed with the SPSS version 21.0 (SPSS Inc, Chicago Illinois) statistical program. The parametric variables were expressed with mean \pm standard deviation, while categorical variables were expressed with the numbers and percentages (\%). The comparisons between two groups was performed with the student's t-test or chi-square test. Pearson correlation analysis was performed to determine the association between the questionnaire scores and AHI and Epworth scores. $\mathrm{p}<0.05$ was regarded as statistically significant.

\section{Results}

Among study participants, 76 had moderate and 72 had severe OSAS. All patients were married. In the moderate OSAS group $18(23.6 \%)$ and the severe OSAS group $21(29.2 \%)$ were housewives. The mean age of the patients in the moderate OSAS group was $35.46 \pm 5.43$ years and in the severe OSAS group it was $40.97 \pm 5.65$ years. Body mass indexes of the moderate and severe OSAS groups were 31.04 $\pm 3.42 \mathrm{~kg} / \mathrm{m}^{2}$ and $33.10 \pm 3.33 \mathrm{~kg} / \mathrm{m}^{2}$, respectively. There were statistically significant differences between severe and moderate OSAS groups regarding both the age and BMI ( $\mathrm{p}<$ 0.001).

In the moderate OSAS group, 25 patients completed primary school, 32 high school, and 14 were university graduates; in the severe OSAS group 19 patients completed primary school, 31 high school, and 26 were university graduates There was not any significant difference between groups regarding the educational status $(\mathrm{p}=0.19)$.

The mean cigarette packet per year smoked by the severe and moderate OSAS groups were $13.61 \pm 11.65$ and $14.60 \pm$ 11.51 , respectively $(\mathrm{p}=0.61)$. The mean Epworth scores of the moderate and severe OSAS groups were $9.52 \pm 3.31$ and $11.45 \pm 3.27$, respectively $(\mathrm{p}=0.001)$. In the severe compared with the moderate OSAS group hypertension (30 vs 7) diabetes mellitus ( 5 vs 0$)$ and coronary artery disease (14 vs 0$)$ were 
TA B L E 3. The results of correlation analysis in all patients.

\begin{tabular}{lcccc} 
& \multicolumn{1}{c}{ FSFI score } & \multicolumn{3}{c}{ SAQLI score } \\
& Correlation coefficient $(\mathbf{r})$ & $\mathbf{p}$ & Correlation coefficient (r) & $\mathbf{p}$ \\
AHI score & -0.189 & 0.014 & -0.214 & 0.003 \\
\hline Epworth score & -0.164 & 0.044 & -0.16 & 0.006 \\
\hline
\end{tabular}

more common.

The BMI did not alter significantly before and after CPAP treatment (in moderate OSAS group $31.04 \pm 3.42 \mathrm{~kg} / \mathrm{m}^{2} \mathrm{vs}$ $30.10 \pm 4.57 \mathrm{~kg} / \mathrm{m}^{2}, \mathrm{p}>0.05$ and in severe OSAS group $33.10 \pm 3.33 \mathrm{~kg} / \mathrm{m}^{2}$ vs $31.28 \pm 4.41 \mathrm{~kg} / \mathrm{m}^{2}, \mathrm{p}>0.05$ ). After treatment, Epworth scores significantly improved in both the moderate $(9.52 \pm 3.30$ vs $8.04 \pm 3.02$, p:0.048) and the severe $(11.45 \pm 3.27$ vs $10.09 \pm 3.62$, p:0.021) OSAS groups.

When the patients were compared for the domains and total FSFI scores, in the pre-treatment period, desire, lubrication, and satisfaction were significantly better in the moderate OSAS group. After treatment, only desire and satisfaction were significantly better in the moderate OSAS group. In both groups, after treatment, all parameters of the FSFI score were significantly better compared with the pretreatment values $p=$ 0.001) (Table 1).

When the patients were compared for the SAQLI scores, in the pre-treatment period, all parameters, daily functioning, social interactions, emotional functioning, and symptoms were significantly better in the moderate OSAS group. In both groups, after treatment, all parameters of the SAQLI score were significantly better compared with the pretreatment values $p=0.001)$ (Table 2).

In correlation analysis performed in all patients, both the FSFI score and SAQLI score showed a significant negative correlation with the AHI and Epworth scores (Table 3).

\section{Discussion}

In this study, we analyzed the sexual health status and life quality of female patients with moderate or severe OSAS before and after treatment with CPAP. Our data indicates that; 1) in the pretreatment period, desire, lubrication and satisfaction were significantly better in the moderate OSAS compared to the severe OSAS group; 2) After 6 months of CPAP treatment, in both OSAS groups all parameters of FSFI and SAQLI scores were improved compared with the pretreatment values; 3) Both FSFI and SAQLI scores showed significant negative correlations with the AHI and Epworth scores. To the best of our knowledge, this is the first study that evaluated the effects of CPAP treatment on female sexual health status.

To date, the data regarding the sexual functions in female OSAS patients is limited. In a prospective study, Koseoglu et al [11] evaluated female OSAS patients with the Sexual Function Questionnaire Version 2 (SFQ-V2) and Epworth Sleepiness Scale and reported that OSAS has negative impacts on sexual function in women independent of age and associated co-morbid diseases. Yilmaz et al [12] compared female OSAS patients with healthy controls and reported that the total FSFI score in the cases with OSAS was significantly worse than the controls. In a prospective study, Jara et al [13] reported that, in severe OSA patients, CPAP treatment was associated with improved sexual quality of life, but not in men. Similarly, Peterson et al [14] also reported that, after 1 year of CPAP treatment, female patients with OSA reported reduced sexual dysfunction. We determined a significant negative correlation between the severity of OSAS and both sexual health and quality of life scores and all scores were improved after 6 months of effective CPAP treatment.

Some previous studies have evaluated the sexual health status and the effects of CPAP treatment in men with OSAS. Cignarelli [15] et al reported that in males with OSAS, there was a correlation between disease severity and sexual dysfunction. The authors also reported an improvement in the negative impact of these conditions on quality of life after CPAP treatment. Pastore et al [16] reported a strong association between OSAS and erectile dysfunction, which improved with sildenafil treatment more than with CPAP alone. Khafagy et al [17] reported that 3 months of nasal CPAP was effective in improving sexual performance and quality of life in males. Pastore et al [16] also reported a correlation between severe OSAS and erectile dysfunction and CPAP was effective in the improvement of the sexual performance of these patients. In a recent interesting study, it was reported that spouse's/partner's engagement was directly related to CPAP adherence and improvement of symptoms, and that CPAP adherence was a mediator of disease-specific health-related quality of life. In this study, a two-way positive interaction was found between the spouse's/partner's engagement and CPAP use [18, 19]. All those data, reported in men, were compatible with our results in women. On the other hand, Celec et al [20] investigated the long-term effects of 1 and 6 months of CPAP treatment on sex hormone concentrations in patients with OSAS and reported that there were no effects of CPAP treatment on testosterone or estradiol levels in OSAS patients of either gender. In that aspect, the pathophysiological mechanisms associated with the sexual health in OSAS patients of both genders should be further investigated. Hormonal status or blood supply to the genital organs may need further studies.

Another interesting point in this study was that the FSFI scores were very homogenous with low standard deviations in both pre-treatment and post-treatment evaluations, which differs from previous reports [21]. This could have been a result of this being a single center study of a homogenous population of pre-menopausal women with a narrow age range.

There are some limitations of this study that should be mentioned. There was a significant difference between patients in the moderate and severe OSAS groups in age and BMI. The differences in FSFI domains in the pre-treatment period between these two groups may be attributed to these factors. The most important factors in the systemic effects of OSAS are 
associated with the oxidative stress parameters. However, in this study, we did not analyze the oxidative stress parameters and we did not analyze the etiological factors of sexual dysfunction in OSAS. We only compared the clinical outcomes. And lastly, absence of an age and gender matched control population of non-OSAS patients is another limitation of the study that prevents us from being able to discuss differences of FSFI scores between OSAS and non-OSAS patients.

In conclusion, in women with severe and moderate OSAS, sexual functions and satisfaction levels as well as quality of life were negatively associated with OSAS severity. Sexual functions and quality of life of women were significantly improved with the effective treatment of OSAS with CPAP. For this reason, in women with OSAS, sexual health status should be evaluated when considering treatment options.

\section{ACKNOWLEDGMENTS}

Thanks to all the peer reviewers and editors for their opinions and suggestions.

\section{CONFLICT OF INTEREST}

No conflict of interest was declared by the authors.

\section{FINANCIAL DISCLOSURE}

The authors declared that this study has received no financial support.

\section{AUTHOR CONTRIBUTIONS}

Dasdemir Ilkhan Gulay: Processing, preparation and writing of the collected information; Critically and intellectually evaluating the content of the article; Analysis of the content of the article; Regulation of the presentation of the information and data in the article; Evaluating the accuracy of the data; Methodology; Statistical evaluation of the article data and the arrangement and interpretation of these data. Celikhisar Hakan: Literature search and analysis; Design and concept of the article; Project administration; Case collection, data gathering and work flow planning; Forming the general lines and framework of the study.

\section{ETHICAL APROVAL}

The study has been reviewed and approved by an Ethical Committee of Bezmialem University (2011- KAEK-42 2019/0502).

\section{REFERENCES}

[1] Passàli D, Tatti P, Toraldo M, et al. OSAS and metabolic diseases: Round Table, 99th SIO National Congress, Bari 2012. Acta Otorhinolaryngol Ital. 2014;34:158-166.

[2] Bonsignore MR, Zito A. Metabolic effects of the obstructive sleep apnea syndrome and cardiovascular risk. Arch Physiol Biochem. 2008;114:255e60-255e60.

[3] Zhuravlev VN, Frank MA, Gomzhin AI. Sexual functions of men with obstructive sleep apnoea syndrome and hypogonadism may improve upon testosterone administration: a pilot study. Andrologia. 2009;41:193-195.

[4] Stannek T, Hürny C, Schoch OD, et al. Factors affecting self-reported sexuality in men with obstructive sleep apnea syndrome. J Sex Med. 2009;6:3415-3424.

[5] Khafagy AH, Khafagy AH. Treatment of obstructive sleep apnoea as a therapeutic modality for associated erectile dysfunction. Int J Clin Pract. 2012;66:1204-1208.

[6] Margel D, Cohen M, Livne PM, et al. Severe, but not mild, obstructive sleep apnea syndrome is associated with erectile dysfunction. Urology. 2004;63:545-549.

[7] Belgü AU, Erdoğan B, San T, et al. The relationship between AHI, Epworth scores and sleep endoscopy in patients with OSAS. Eur Arch Otorhinolaryngol. 2015;272:241-245.

[8] Doneh B. Epworth Sleepiness Scale. Occup Med (Lond). 2015;65:508.

[9] Meston CM, Freihart BK, Handy AB, et al. Scoring and Interpretation of the FSFI: What can be Learned From 20 Years of use? J Sex Med. 2020;17:17-25.

[10] Flemons WW, Reimer MA. Development of a disease-specific healthrelated quality of life questionnaire for sleep apnea. Am J Respir Crit Care Med. 1998;158:494-503.

[11] Köseoğlu N, Köseoğlu H, Itil O, et al. Sexual function status in women with obstructive sleep apnea syndrome. J Sex Med. 2007;4:1352-1357.

[12] Yilmaz Z, Sirinocak PB, Voyvoda B, et al. Sexual Dysfunction in Premenopausal Women with Obstructive Sleep Apnea. Urol J. 2017;14:5051-5056.

[13] Jara SM, Hopp ML, Weaver EMC. Association of Continuous Positive Airway Pressure Treatment With Sexual Quality of Life in Patients With Sleep Apnea: Follow-up Study of a Randomized Clinical Trial. JAMA Otolaryngol Head Neck Surg. 2018;144:587-593.

[14] Petersen M, Kristensen E, Berg S, et al. Long-term effects of continuous positive airway pressure treatment on sexuality in female patients with obstructive sleep apnea. Sex Med. 2013;1:62-68.

[15] Cignarelli A, Castellana M, Castellana G, et al. Effects of CPAP on Testosterone Levels in Patients With Obstructive Sleep Apnea: A MetaAnalysis Study. Front. Endocrinol. 2019;10:551.

[16] Pastore AL, Palleschi G, Ripoli A, et al. Severe obstructive sleep apnoea syndrome and erectile dysfunction: a prospective randomised study to compare sildenafil vs. nasal continuous positive airway pressure. Int $\mathrm{J}$ Clin Pract. 2014;68:995-1000.

[17] Khafagy AH, Khafagy AH. Treatment of obstructive sleep apnoea as a therapeutic modality for associated erectile dysfunction. Int J Clin Pract. 2012;66:1204-1208.

[18] Gentina T, Bailly S, Jounieaux F, et al. Marital quality, partner's engagement and continuous positive airway pressure adherence in obstructive sleep apnea. Sleep Med. 2019;55:56-61.

[19] Taskin U, Yigit O, Acioglu E, et al. Erectile dysfunction in severe sleep apnea patients and response to CPAP. Int J Impot Res. 2010;22:134-139.

[20] Celec P, Mucska I, Ostatníková D, et al. Testosterone and estradiol are not affected in male and female patients with obstructive sleep apnea treated with continuous positive airway pressure. J Endocrinol Invest. 2014;37:912.

[21] Chedraui P, Perez-Lopez FR, San Miguel G, et al. Assessment of sexuality among middle-aged women using the Female Sexual Function Index. Climacteric. 2009;12:213-221.

How to cite this article: Dasdemir Ilkhan Gulay, Celikhisar Hakan. The Effect of Positive Airway Pressure Therapy on Sexual Life and Quality of Life in Women with Moderate or Severe Obstructive Sleep Apnea Syndrome. Signa Vitae. 2021;17(1):128-132. doi:10.22514/sv.2020.16.0069. 